\title{
A New Method for the Measurement of Low Speed Using a Multiple-Electrode Capacitive Sensor
}

\author{
Xiujun Li and Gerard C. M. Meijer, Member, IEEE
}

\begin{abstract}
A novel method for the measurement of low speeds by using a low-cost capacitive sensor is presented. Its main advantages are its simplicity, low cost and the very low speed (as low as $0.02 \mathrm{rad} / \mathrm{s}$ ), which can be measured with a relatively high resolution in a short measurement time. The multiple capacitors, whose values are piece-wise linearly related to the position in a periodic way, are used as the sensing elements of the low-speed sensor. A capacitance-controlled oscillator is used in the processing circuit to convert the capacitance to a period-modulated signal which can be read directly out by a microcontroller. An algorithm, which is used to calculate the speed from the measured periods, either eliminates or strongly reduces the influence of most errors. The measurement time is relatively short for low speeds. Meanwhile, the direction of movement can be indicated.
\end{abstract}

Index Terms-Low speed, sensor, speed measurement, velocity measurement.

\section{INTRODUCTION}

$\mathbf{M}$ ANY DIGITAL measurements of speed which use the spatial-filter method [1]-[3] have been presented. In this kind of measurement, it will take a relatively long time to perform one measurement of low speed. A simple alternative and relatively faster method for the measurement of very low speed is to use a potentiometer as the sensing element [4]. When using this method, an accuracy of $0.8 \%$ has been obtained for a speed of $0.02 \mathrm{rad} / \mathrm{s}$ in a measurement time of $200 \mathrm{~ms}$. However, since the sliding contact is directly in contact with the resistive element of the potentiometer, the long-term stability is bad and there is hysteresis when the speed and its direction are changed. In [5], a capacitive speed sensor is presented. This sensor is only suitable to the measurement of high and middle speeds (larger than a few $\mathrm{rad} / \mathrm{s})$.

In this paper, a new method for the measurement of low speed is presented. Multiple capacitors, with values which are almost linearly related to the position within a certain range are used as the sensing elements of the speed sensor. A highly linear capacitance-controlled oscillator is used in the processing circuit to convert the capacitance to a period-modulated signal which can directly be read out by a microcontroller. The algorithm used to calculate the speed either eliminates or strongly reduces the influence of the many nonidealities, such as delay time, offset voltage and bias current of the op amp.

Manuscript received June 20, 1996; revised October 1, 1996.

The authors are with the Department of Electrical Engineering, Delft University of Technology, 2628 CD Delft, The Netherlands.

Publisher Item Identifier S 0018-9456(97)01592-1.

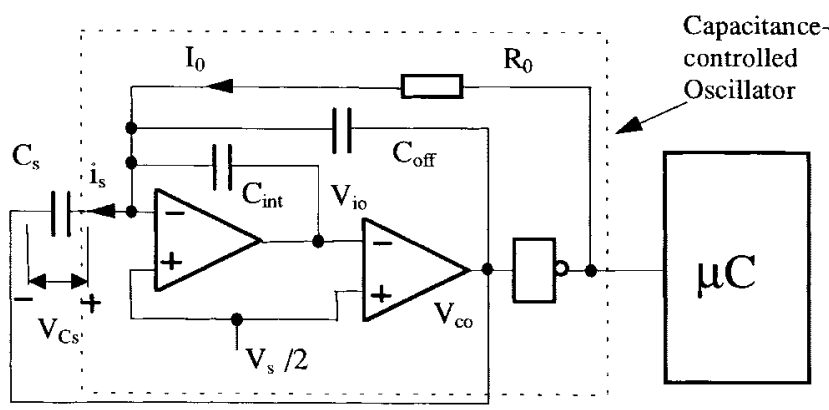

Fig. 1. An overview of the measurement system for low speed.

\section{MEASUREMENT PRINCIPLE}

The three-layered electrode structure of a capacitor, which consists of two fixed electrodes and one moveable electrode (see Fig. 3), is often used as the sensing element for the measurement of physical quantities. The two fixed parallel electrodes form a capacitor and the grounding moveable electrode shields the electric field between two fixed electrodes. The value of the capacitor between two fixed electrodes is changed when the position of the moveable electrode changes. If the value of a capacitor varies linearly in response to the position of the moveable electrode, it can be used as the sensing element for measurement of the speed. The relation between the speed $\vartheta_{n}$ to be measured and the capacitance can be described as

$$
\vartheta_{n}=\frac{\mathrm{d} x}{\mathrm{~d} t}=\frac{\partial C_{\mathrm{s}} / \partial t}{\partial C_{\mathrm{s}} / \partial x}=\frac{1}{K} \cdot \frac{\partial C_{\mathrm{s}}}{\partial t}
$$

where $K$ is a constant defined by the structure of the capacitive sensing element, $x$ represents the (angular) position and $t$ represents time.

Fig. 1 shows an overview of the measurement system for low speed. The sensing element for the speed is a capacitor $C_{\mathrm{S}}$, whose value linearly varies in response to the position of moveable electrode.

The time-variant capacitance $C_{\mathrm{s}}$ is continuously converted into period-modulated signals by using a capacitancecontrolled oscillator which generates a square-wave output signal. This period-modulated signal can directly be read out and processed by a microcontroller. Fig. 2 shows the waveform of voltage $V_{\text {io }}$ at the integrator output of the oscillator, for the case where the moveable electrode of the capacitive sensing element is moving linearly with respect to the fixed electrodes. 


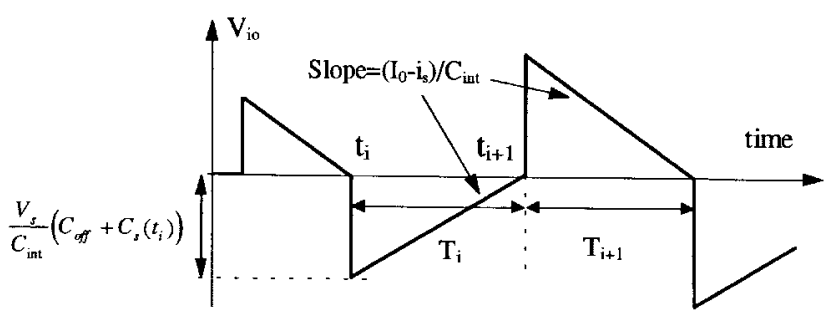

Fig. 2. The waveform of $V_{\mathrm{io}}$ at the output of the integrator.

According to the principle of the oscillator, for a constant speed, two adjacent charge and discharge time intervals $T_{i}$ and $T_{i+1}$ amount to

$$
\begin{gathered}
T_{i}=\frac{V_{\mathrm{s}}\left[C_{\mathrm{s}}\left(t_{i}\right)+C_{\mathrm{off}}\right]}{I_{0}-i_{\mathrm{s}}} \\
T_{i+1}=\frac{V_{\mathrm{s}}\left[C_{\mathrm{s}}\left(t_{i+1}\right)+C_{\mathrm{off}}\right]}{I_{0}-i_{\mathrm{s}}}
\end{gathered}
$$

where $V_{\mathrm{s}}$ is the power supply voltage and $i_{\mathrm{s}}$ is a current which is caused by the change of the capacitance $C_{\mathrm{s}}$ with time.

For the current $i_{\mathrm{s}}$ it holds that

$$
i_{\mathrm{s}}= \pm \frac{V_{\mathrm{s}}}{2} \frac{\partial C_{\mathrm{s}}(t)}{\partial t}= \pm \frac{V_{\mathrm{s}}}{2} K \vartheta_{n} .
$$

For the constant speed, the relation between capacitances $C_{\mathrm{s}}\left(t_{i}\right)$ at moment $t_{i}$ and $C_{\mathrm{s}}\left(t_{i+1}\right)$ at moment $t_{i+1}$ is represented by

$$
C_{\mathrm{s}}\left(t_{i+1}\right)=C_{\mathrm{s}}\left(t_{i}\right)+\frac{\partial C_{\mathrm{s}}}{\partial t} T_{i}
$$

From (2)-(5), the average speed $\vartheta_{n}$ over time interval $T_{i}+$ $T_{i+1}$ is obtained, it is

$$
\vartheta_{n}=\frac{1}{K R_{0}} \cdot\left(1-\frac{T_{i}}{T_{i+1}}\right) .
$$

where factor $1 / K R_{0}$ is determined by the structure of capacitive sensing element and a resistance. By measuring two adjacent half-periods $T_{i}$ and $T_{i+1}$, and using (6), the average speed over time interval $T_{i}+T_{i+1}$ can be measured. However, this measuring method has the following disadvantages:

1) Errors caused by the delay time $t_{d}$ of the oscillator.

2) Serious influence of offset voltage and bias current of the op amp's in the oscillator.

3) Large sampling noise due to very short measuring time $\left(T_{i}+T_{i+1}\right)$.

These disadvantages can be overcome by measuring many full periods. Supposing $T_{s 1}$ and $T_{s 2}$ represent two adjacent measurement time intervals for $N$ full periods of the oscillator, respectively, according to

$$
T_{s 1}=\sum_{i=1}^{2 N} T_{i}, \quad T_{s 2}=\sum_{i=2 N+1}^{4 N} T_{i}
$$

then after some calculations, for the measured average speed $\vartheta_{n}$ over time $T_{s 1}+T_{s 2}$, it is found that:

$$
\vartheta_{n}=\frac{1}{K R_{0}} \cdot\left[1-\left(\frac{T_{s 1}}{T_{s 2}}\right)^{1 / 2 N}\right] \text {. }
$$

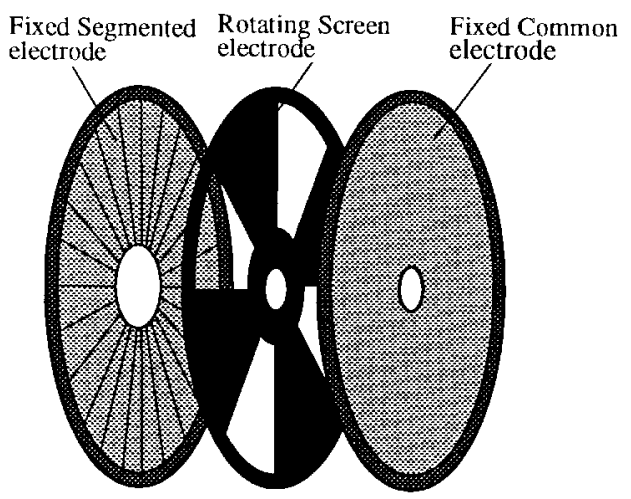

Fig. 3. A simplified structure of the capacitive sensing element of the angular-speed transducer.

By measuring the two adjacent time intervals $\left(T_{s 1}\right.$ and $\left.T_{s 2}\right)$ for $N$ full periods, the speed is calculated with formula (8). Meanwhile, the sign of the result of formula (8) indicates the direction of movement.

\section{StRucture OF THE SENSING ElEMENT AND MEasurement Method}

\section{A. Sensing Element}

A simplified structure of the capacitive sensing element used to measure angular speed is shown in Fig. 3 [5]. It consists mainly of three parts: a rotating screen electrode with four identical windows which are equidistant, a fixed common electrode which is a single conductor, and a fixed segmented electrode with 24 segments. For the segmented electrode, four segments in quadrature positions were connected in parallel, which results in six capacitors $\left(C_{1}, C_{2}, \cdots, C_{6}\right)$ between the segmented electrode and common electrode. This parallel connection of quadrature elements significantly reduces the influences of mechanical errors, such as nonflatness of the electrode surfaces, eccentricity, and obliqueness of the electrodes. The six capacitors are used for the low-speed measurement.

The rotating screen electrode partly shields the segmented electrode from the common electrode and so decreases the total capacitance between the two fixed electrodes. For the structure shown in Fig. 3, by selectively connecting segments, the relations between the six capacitances and position can be obtained (see Fig. 4). In this figure, $C_{1}^{\prime}=C_{1}+C_{2}+C_{3}$, $C_{3}^{\prime}=C_{3}+C_{4}+C_{5}$ and $C_{5}^{\prime}=C_{5}+C_{6}+C_{1}$, and their linear parts, which are indicated by the bold polygon, are used for the low-speed measurement. This method reduces the influence of the regions of the nonlinear electric field, see the thin parts of the curves in Fig. 4.

\section{B. Measurement Method}

In the measurement of speed, first, the capacitor which is used as the sensing element must be known, that is, the one which has a linear relation with the position during the measurement. Second, this capacitance is measured twice $\left(T_{s 1}\right.$ and $T_{S 2}$ ). Fig. 5 shows the measurement process for low speed.

Where factor $\alpha$, whose value is 1 or -1 , indicates the direction of movement. $T_{1}^{\prime}, T_{3}^{\prime}$, and $T_{5}^{\prime}$ are, respectively, time 


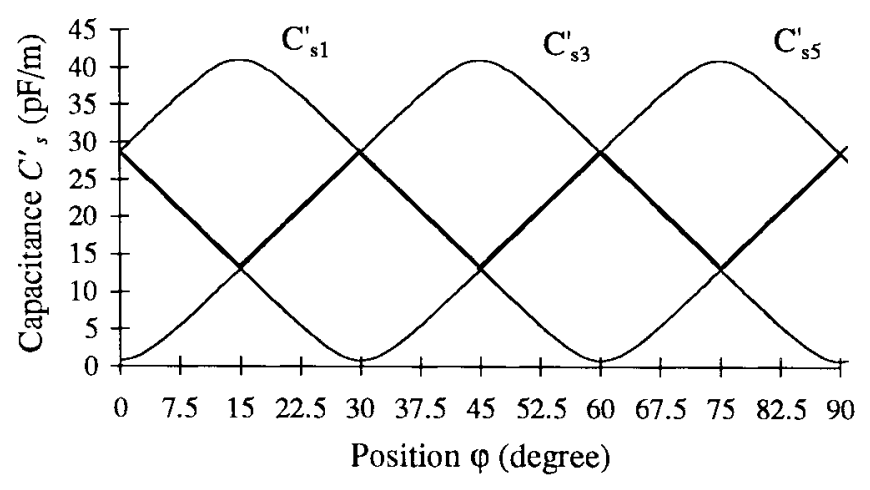

Fig. 4. The capacitances as a function of the angular position.

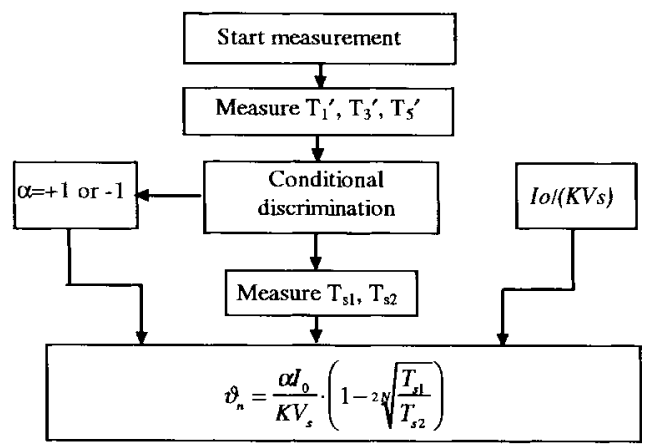

Fig. 5. The measurement process for low speed.

TABLE I

The CONDITIONAL Discriminations

\begin{tabular}{c|c|c}
\hline Discrimination & $C_{5}$ & $\alpha$ \\
\hline$T_{5}{ }^{\prime}>T_{1}>T_{3}{ }^{\prime}$ & $C_{1}^{\prime}$ & +1 \\
\hline$T_{1}{ }^{\prime}>T_{5}>T_{3}{ }^{\prime}$ & $C_{5}{ }^{\prime}$ & -1 \\
\hline$T_{1}>T_{3}>T_{5}{ }^{\prime}$ & $C_{3}{ }^{\prime}$ & +1 \\
\hline$T_{3}{ }^{\prime}>T_{1}>T_{5}{ }^{\prime}$ & $C_{1}{ }^{\prime}$ & -1 \\
\hline$T_{3}{ }^{\prime}>T_{5}>T_{1}^{\prime}$ & $C_{5}{ }^{\prime}$ & +1 \\
\hline$T_{5}>T_{3}>T_{1}^{\prime}$ & $C_{3}^{\prime}$ & -1 \\
\hline
\end{tabular}

intervals for $N$ full periods of the oscillator that correspond to capacitances $C_{1}^{\prime}, C_{3}^{\prime}$, and $C_{5}^{\prime}$.

The conditional discriminations are listed in Table I. This table follows from Fig. 4. $C_{\mathrm{s}}$ represents the capacitance whose linear part, relative to position, is used as the sensing element for the measurement of low speed.

\section{Limitation of THE MEASUREMENT RANGE}

\section{A. Minimum Measurable Speed}

The minimum measurable speed is mainly limited by the choice of the measurement time $T_{m}$, the maximum and minimum capacitances $\left(C_{i, \max }, C_{i, \min }\right)$ between the common electrode and one segment electrode, and the resolution of the signal processor. Depending on the measurement process described in Fig. 5, the total time for one successive measure- ment is

$$
T_{m}=T_{m}^{\prime}+T_{s 1}+T_{s 2}
$$

Where $T_{m}^{\prime}$ is the measurement time for measuring capacitances $C_{1}^{\prime}, C_{3}^{\prime}$ and $C_{5}^{\prime}$, in order to be able to perform the conditional discrimination. Then the minimum measurable speed $\vartheta_{n \text { min }}$ is represented as

$$
\vartheta_{n \text { min }} \geq \frac{\delta C_{\mathrm{re}}}{12\left(C_{i, \max }-C_{i, \min }\right)\left(T_{s 1}+T_{s 2}\right)}
$$

where $\delta C_{\mathrm{re}}$ is the resolution of the sensor system for capacitance measurement, which includes the sampling noise of the microcontroller. Here, the unit of $\vartheta_{n \text { min }}$ is $\mathrm{rad} / \mathrm{s}$.

For example, if $\delta C_{\text {re }}=50 \mathrm{aF}, C_{i, \max }-C_{i, \min }=0.15 \mathrm{pF}$ and $T_{s 1}+T_{s 2}=10 \mathrm{~ms}$, Then $\vartheta_{n \text { min }} \geq 2.8 \times 10^{-3} \mathrm{rad} / \mathrm{s}$.

\section{B. Maximum Measurable Speed}

The maximum measurable speed is mainly limited by the measurement time $T_{m}$

$$
\vartheta_{n \max } \leq 1 / 36 T_{m}
$$

Here, the unit of $\vartheta_{n \max }$ is $\mathrm{rad} / \mathrm{s}$. For example, if $T_{m}=10$ ms, Then $\vartheta_{n \max } \leq 2.8 \mathrm{rad} / \mathrm{s}$.

\section{EXPERIMENTAL RESUlTS}

An angular-speed sensing element based on the structure described in Fig. 3 has been built and tested. The common electrode and segmented electrode were constructed using simple, printed circuit-board technology. The capacitance between each of the nonshielded segments and the common electrode amounts to about $0.15 \mathrm{pF}$. Guarding electrodes surrounding the segmented electrode and common electrode were used to reduce the influence of the electric-field bending in radial directions and of electromagnetic interference. The rotating electrode is grounded by a sliding contact. All the electrodes are mounted in a metal housing, which is connected to ground and shields against external interference. The five time intervals, $T_{1}^{\prime}, T_{3}^{\prime}, T_{5}^{\prime}, T_{s 1}$, and $T_{s 2}$, were measured in a total measurement time of about $100 \mathrm{~ms}$. The factor $1 / K R_{0}$ was used as a constant. The noise of the signal processing circuit, including the noise of the signal processor, the sampling noise of the microcontroller, and the noise of the sensing element, was measured under identical conditions in a fixed position of the rotating electrode $(=0)$ and a measurement time of about $100 \mathrm{~ms}$. The standard deviation of this noise amounts to $3 \times 10^{-4} \mathrm{rad} / \mathrm{s}$.

The relation of the minimum measurable speed to the measurement time was measured in accordance with (10) under identical conditions in a fixed position of the rotating electrode $(=0)$. The relation of the maximum measurable speed to the measurement time was calculated from (11). The two relations are shown in Fig. 6.

From this figure we see that the increase of the measurement time results in the decrease of both maximum and minimum measurable speeds, as was to be expected in view of (10) and (11). 


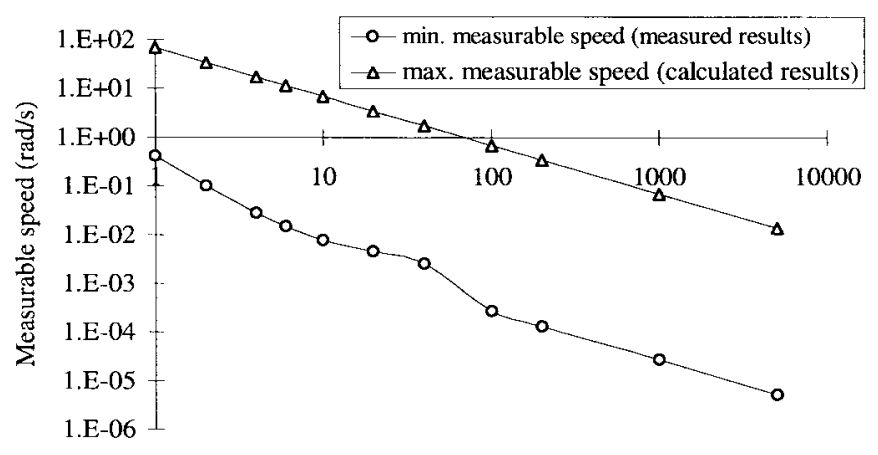

The time for one complete measurement (ms)

Fig. 6. The relations between the measurable speeds and the measurement time.

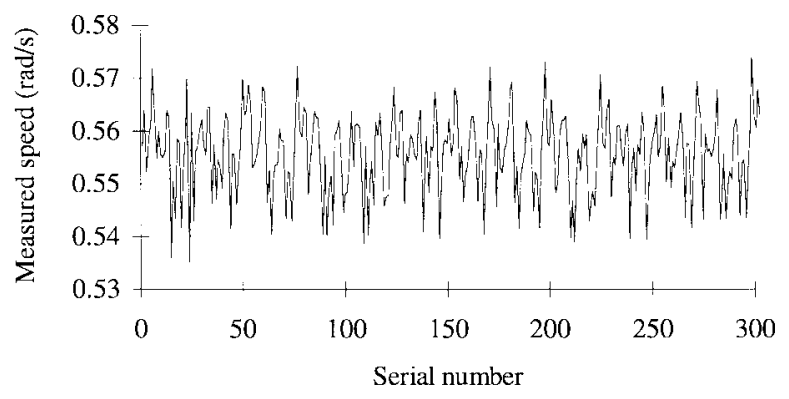

Fig. 7. The measured result of a series of 300 measurements performed under a constant speed $(=0.55 \mathrm{rad} / \mathrm{s})$. Each measurement takes about $50 \mathrm{~ms}$.

For a constant speed of about $0.55 \mathrm{rad} / \mathrm{s}$, the standard deviation was measured and amounted to $7.5 \times 10^{-3} \mathrm{rad} / \mathrm{s}$ (see Fig. 7). A measurement time of $50 \mathrm{~ms}$ was used to ensure that the measured speed of $0.55 \mathrm{rad} / \mathrm{s}$ was between the maximum and minimum measurable speeds. The deviation originates mainly from the uncertainty of the speed source, the mechanical deviation of the sensing element, the noise of the signal processor, and the sampling noise of the microcontroller. Compare the above deviation with the measured standard deviation of the signal processing circuit $\left(3 \times 10^{-4}\right)$, it is concluded that the uncertainty of the speed source and the mechanical deviation of the sensing element are the main sources of the deviation of $0.55 \mathrm{rad} / \mathrm{s}$.

\section{CONCLUSIONS}

A novel low-cost capacitive sensor for the measurement of low speed has been presented. A simple and accurate multiplecapacitive sensing element with three parallel discs has been used for the measurement of low speed, as well as for the accurate measurement of angular position. The applied algorithm for the measurement of low speed either eliminates or strongly reduces the influences of many systematic nonidealities of the sensing element and the signal processor in a very effective way. A microcontroller is used to measure time intervals, to calculate speed and to communicate with the outside digital world. A prototype of the capacitive speed sensor has been built with low-cost material. For a constant speed of 0.55 $\mathrm{rad} / \mathrm{s}$, the measured standard deviation amounts to $7.5 \times 10^{-3}$ $\mathrm{rad} / \mathrm{s}$ in a measurement time of $50 \mathrm{~ms}$. Compared to existing speed sensors, the main advantages of this novel capacitive low-speed sensor are its simplicity, low cost, and the very low speed that can be measured with a relatively high resolution in a short measurement time. The sensor system also indicates the direction of rotation.

\section{REFERENCES}

[1] R. Bonert, "Design of a high-performance digital tachometer with a microcontroller," IEEE Trans. Instrum. Meas., vol. 38, pp. 1104-1108, Dec. 1989.

[2] Y. Ameari and I. Masuda, "Velocity sense detection based on the spatial filter method," IEEE Trans. Instrum. Meas., vol. 39, pp. 649-652, Dec. 1990.

[3] C. D. diCenzo, B. Szabados, and N. K. Sinha, "Digital measurement of angular velocity for instrumentation and control," IEEE Trans. Ind. Electron. Contr. Instrum., vol. IECI-23, pp. 83-86, Feb. 1976.

[4] W. Ahmad and M. Ahmad, "A potentiometric transducer for the measurement of very low speeds," IEEE Trans. Instrum. Meas., vol. 34, pp. 470-471, Sept. 1985.

[5] G. W. de Jong, "Smart capacitive sensors (physical, geometrical, and electronic aspects)," Ph.D. dissertation, Dept. Elect. Eng., Delft Univ. of Technology, 1994.

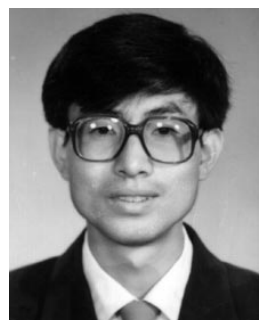

Xiujun Li was born in Tianjin, China, on February 19,1963 . He received the B.S. degree in physics and the M.S. degree in electrical engineering from Nankai University, Tianjin, in 1983 and 1986, respectively. He is now pursuing the Ph.D. degree in electrical engineering at Delft University of Technology, Delft, The Netherlands.

He joined the Department of Electronic Science, Nankai University, in 1986. His research interests are in the area of the smart capacitive sensor and signal processing.

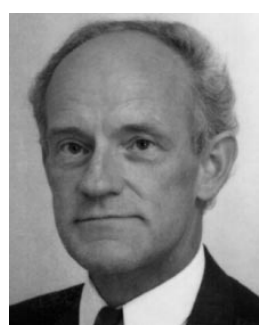

Gerard C. M. Meijer (M'94) was born in Wateringen, The Netherlands, on June 28, 1945. He received the ingenieurs (M.S.) and Ph.D. degrees in electrical engineering from the Delft University of Technology, Delft, The Netherlands, in 1972 and 1982, respectively.

Since 1972 he has been with the Laboratory of Electronics, Delft University of Technology, where he is an Associate Professor, engaged in research and teaching on analog IC's. In 1984 and part-time during 1985-1987 he was with the Delft Instruments Company, Delft, where he was involved in the development of industrial level gauges and temperature transducers.

Dr. Meijer is a member of the Netherlands Society for Radio and Electronics. 